\title{
Solutions of scalar mean profiles close to gas-liquid interfaces under turbulent free slip motion
}

\author{
H. E. Schulz ${ }^{1,2}$ \& B. B. Gonçalves ${ }^{1}$ \\ ${ }^{1}$ Department of Hydraulics and Sanitary Engineering, \\ University of São Paulo, Brazil \\ ${ }^{2}$ Nucleus of Thermal Sciences and Engineering, \\ University of São Paulo, Brazil
}

\begin{abstract}
Mean profiles of scalar properties close to moving gas-liquid interfaces subjected to turbulence are quantified using Random Square Waves (RSW). The condition of stationary turbulent transfer allows reducing the third order nonlinear governing equation successively to a second order and to a first order equation, which admits theoretical integration, furnishing the set of solutions analyzed in the present study. It is shown that different solutions may apply to different parts of the calculation domain (physical domain). It is also shown that the stationary problem admits a linear profile close to the gas-liquid interface, while a nonlinear function describes the mean properties apart from the interface.
\end{abstract}

Keywords: turbulence, interfacial transfers, nonlinear equations, RSW method.

\section{Introduction}

The transfer of scalar properties through turbulent interfaces is a theme of interest in different fields of applied sciences. Examples are the gas transfer across airwater interfaces in geophysical flows as lakes, rivers, estuaries and oceans; or the transfer of chemical species between phases in unit operations of industrial procedures, like the manufacture of petrochemicals, the production of pharmaceuticals, and the sewage treatment. Different aspects of interfacial transfer may be found in Asher et al. [1], Demars and Manson [2], Nguyen and Tan [3], Krah [4], among others. 
Due to its complexity, turbulent transfer is the theme of theoretical studies, in which models for statistical properties are proposed, and evolutions of mean proprieties in space and time are quantified (see [5], for example). Such proposals consider the necessary correspondence between predictions and physical reality. Experiments are thus relevant to consubstantiate theoretical propositions. See for example $[6,7]$. Measurements of scalar variables close to interfaces, under both the free slip or the no slip condition, are reasonably frequent in the literature, providing support for conceptual discussions. Examples are found in [8, 9]. The transfer of scalar properties through turbulent free slip interfaces is studied here. Because measurements on oxygen transfer were used for comparisons (Janzen [10]), the discussions consider the concentration of low soluble gases in liquids.

For air-water gas transfer, the LIF methodology (Laser Induced Fluorescence), presented by Wolff et al. [11], provides detailed mean gas concentration profiles. But a flattening may occur at interfaces, described by Duke and Hanratty [12] as: "An unexpected behavior occurs in the region close to the interface, where the concentration appears constant. This is where one would expect to see the largest value of the concentration gradient". Optical effects were suggested as possible causes of this constancy. Münsterer et al. [13] mentioned a possible optical blurring or a real physical effect. Woodrow and Duke [14], assuming the optical blurring, used a corrective procedure: the largest measured gradient was selected, and a straight line was drawn for the profile from the point of largest gradient until the interface. Janzen [10] also used this correction to obtain mean concentration and fluctuation profiles, which, together with concomitant velocity values, furnished turbulent mass fluxes (Schulz and Janzen [8], and Janzen et al. [7]). Falkenroth [15] described details of the mentioned blurring, presenting more precise techniques for the largest gradient criterion. The interfacial region thus presents characteristics still not totally explained. Eventual "real flattening" or "linear extrapolations" still seem to need more detailed attention.

High resolution concomitant measurements of interfacial scalar and velocity fields remain difficult, and experimental information of higher order statistical variables is unavailable in the present. Numerical tools are then used, generating mean profiles and statistical parameters of the variable under study. For example, Calmet and Magnaudet [16] studied the mass transfer at air-water interfaces for high Schmidt numbers using a LES (Large Eddy Simulation) scheme, while Hasegawa and Kasagi [17, 18] quantified the effect of different Schmidt numbers using a hybrid LES/DNS (Direct Numerical Simulation) scheme. Calmet and Magnaudet [19] and Magnaudet and Calmet [20] also quantified mass transfer for several Schmidt numbers, arguing that LES allows using higher Reynolds numbers. There is no flattening of numerical profiles, that is, no interfacial blurring is reproduced. Conclusions of numerical studies at air-water interfaces are used in review texts, such as Ishihara et al. [21].

Classical concepts of statistical turbulence are found in texts like Hinze [22], Brodkey [23], Monin and Yaglom [24], in which equations of statistical fluid mechanics and basic solved cases, with their hypotheses, are presented. General solutions are still not available, and the models are described mentioning their uses and limits. The hypothesis of Boussinesq, which replaces products of fluctuating 
properties by a gradient of a mean variable multiplying a convenient coefficient (see [24], for example), is perhaps the most known approximation in this field. The coefficient is written as a function of the simulated flow. Proposals are described in Poper [25], for example. The same coefficient may be used for different products that arise from the statistical procedures, multiplied by corrective factors. The statistical procedures follow, briefly described, two steps: 1) the set of equations is formed; and 2) additional "a posteriori" equations are supplied to "close" the set (originally "open").

For turbulent scalar transport, Schulz and Janzen [8] and Schulz et al. [26, 27] used a "random square waves" (RSW) methodology, applied to one-dimensional (1D) mass transport. For this 1D case, four functions were defined to "adjust" a square random signal to the original turbulent record. Equations were then built using these "a priori" functions, and all the products of fluctuations are combinations of them. Using a so called constant "reduction function, $\alpha$ ", the 1D scalar transport is governed by a non-linear third order differential equation. Transient cases were studied numerically by Lopes Jr. and Schulz [28] and Gonçalves and Schulz [29], discussing discontinuities of the higher derivatives.

This study furnishes mean scalar profiles at turbulent interfaces for permanent cases of the 1D RSW equation, using constant "reduction functions". It allowed: i) obtaining theoretical solutions for adequate values of the reduction function; ii) showing that the profiles alter for different values of the reduction function.

\section{RSW equations for $1 D$ turbulent transfer at interfaces}

Schulz et al. [26] proposed eqn (1) for the mean concentration of a scalar, $n$, at interfacial regions. $n$ was taken as the gas concentration in water.

$$
\begin{aligned}
& A\left[2 A n(1-n)+\frac{(1-2 n)}{2}\right] \frac{d^{3} n}{d z^{3}} \frac{d n}{d z^{*}}+A\left\{-\left[2 A n(1-n)+\frac{(1-2 n)}{2}\right] \frac{d^{2} n}{d z^{*}}+\right. \\
& \left.+\frac{\kappa(1-n)[2 n(A-1)+1]}{2}+\frac{\{1+2 A[A(1-2 n)-1]\}}{A}\left(\frac{d n}{d z^{*}}\right)^{2}\right\} \frac{d^{2} n}{d z^{2}}+ \\
& +\kappa\{(A-1)(1-n)-A[A(1-2 n)-(3 / 2-2 n)]\}\left(\frac{d n}{d z^{*}}\right)^{2}=0
\end{aligned}
$$

$z^{*}=z / E$ where $z$ is the axis normal to the interface, and $E$ is the boundary layer thickness. Schulz et al. [26, 27] used $E$ defined for $0 \leq n \leq 1$, but here it is defined for $0.01 \leq n \leq 1 . \kappa$ is a nondimensional transfer coefficient. $A=(1-\alpha)$, where $\alpha$ is the reduction function, subjected to $0<\alpha<1$, and assumed constant for first analyses. Higher $\alpha$ imply higher diffusive effects, related to the agitation of the liquid. Numerical solutions for transient cases are found in Schulz et al. [26, 27], Lopes Jr. and Schulz [28], Gonçalves and Schulz [29] and Gonçalves [30]. Eqn (2) is an equivalent integrated second order equation obtained for eqn (1), described by Souza et al. [31]. $\Omega_{1}$ is the integration constant. The set $\mathrm{d}^{3} n / \mathrm{d} z^{*}, \mathrm{~d}^{2} n / \mathrm{d} z^{* 2}, \mathrm{~d} n / \mathrm{d} z^{*}$, $n$ is replaced by $\mathrm{d}^{2} n / \mathrm{d} z^{* 2}, \mathrm{~d} n / \mathrm{d} z^{*}, n$ and $\int n \mathrm{~d} z^{*}$, but eqn (2) is simpler than eqn (1). 


$$
\begin{aligned}
& -A(1-n)\left[A n+\frac{(1-2 n)}{2}\right]+(1-A)\left[\frac{1}{\kappa} \frac{d n}{d z^{*}}-z^{*}+\int n d z^{*}+\Omega_{1}\right] \frac{d n}{d z^{*}}= \\
& =-\frac{A}{2 \kappa}[4 A n(1-n)+(1-2 n)] \frac{d^{2} n}{d z^{* 2}}
\end{aligned}
$$

Appendix A shows how eqn (2) is obtained from the original formulation. Eqns (1) and (2) have at least two elementary solutions, in the form:

$$
\begin{gathered}
n=\text { Constant } \\
n=-\Omega_{5} z *+\Omega_{4}
\end{gathered}
$$

Solution (3a) satisfies eqn (1), and eqn (2) furnishes additional information that restricts this solution to Constant $=1 /[2(1-A)]$. Solution (3b) satisfies eqn (1) for $A=1 / 2$. In this case, eqn (2) shows that, because for $z^{*}=0$ we have $n=1.0$, then $A=1 / 2$ implies in $\Omega_{5}=\Omega_{1} \kappa$, and $\Omega_{4}=1.0$. A constant $n$ (eqn 3a) is not viewed as viable for practical purposes. But the linear profile with negative slope (eqn $3 \mathrm{~b}$ ) is a usual approximation, being one of the first attempts for interfacial transfers (see Lewis and Withman [32], for example). As mentioned, also recent studies use such linear extrapolations close to interfaces (Falkenroth [15]). But it is not realistic to use it for the whole boundary layer, although numerical solutions of eqn (1) show this theoretical possibility. Fig. 1, adapted from Schulz et al. [26] shows the trend to linear profiles for lower values of $z^{*}$ obtained with eqn (1), and also the mentioned straight line covering the whole domain.

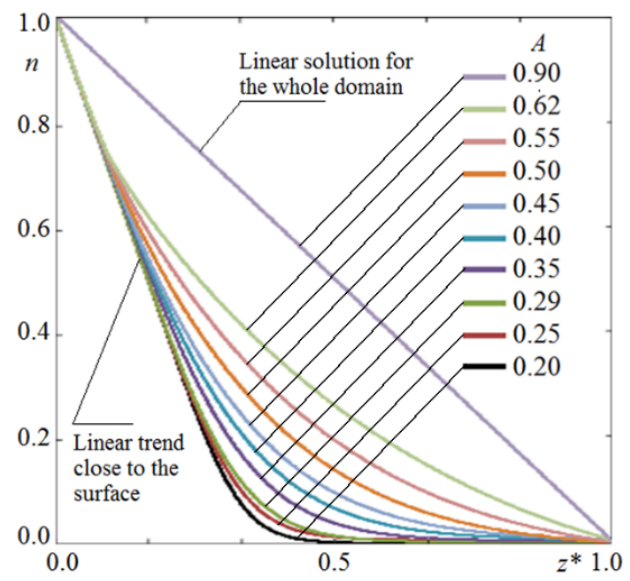

Figure 1: $n$ profiles (scalar concentration profiles) for $0.20 \leq A \leq 0.90 . A=0.90$ plots a linear profile for the whole domain. Adapted from Schulz et al. [26].

Fig. 1 suggests that linear solutions hold close to the interface, while nonlinear functions hold far from the interface. The permanent case is used here to verify these trends. First numerical profiles of $n$ comparing eqns (1) and (2) were presented by Souza et al. [31], and an exhaustive comparison was made by Gonçalves [30], showing similar results and details of both formulations. Gonçalves [30] obtained eqn (4) for the permanent (stationary) case of eqn (2): 


$$
(1-A)\left[\frac{d n}{d z^{*}}+\Omega_{2}\right] \frac{d n}{d z^{*}}=-\frac{A}{2}[4 A n(1-n)+(1-2 n)] \frac{d^{2} n}{d z^{* 2}}
$$

$\Omega_{2}$ is an integration constant. Appendix B shows the derivation of eqn (4). It admits different forms of integration, one of them given in Gonçalves [30], as:

$$
\frac{d n}{d z^{*}}=-\Omega_{2}+\Omega_{3} \exp \left\{\frac{2(1-A)}{A \sqrt{4 A^{2}+1}} \operatorname{Atanh}\left[\frac{\mathrm{A}(2-4 \mathrm{n})-1}{\sqrt{4 A^{2}+1}}\right]\right\}
$$

$\Omega_{3}$ is an integration constant. The RSW method thus allowed reducing the $1 \mathrm{D}$ interfacial transport problem to a first order differential equation. In the present study a second form of integration of eqn (4) was used, as shown in section 3, leading to a set of explicit solutions for the permanent $n$ profiles.

\section{Solutions for the permanent one-dimensional case}

Eqns (3a) and (3b) are of course also solutions of the permanent case given by eqn (4). Using the constants of the permanent problem, eqn (3b) is replaced by:

$$
n=-\Omega_{2} z^{*}+\Omega_{4}
$$

$\Omega_{4}$ is an integration constant. A first nonlinear solution of eqn (5) is:

$$
\begin{gathered}
\frac{1}{\exp \left[\theta_{1} \operatorname{Atanh}\left(\theta_{2}-\theta_{3} n\right)\right]+1}+\frac{1}{1+\theta_{5}} \ln \frac{\exp \left[\theta_{1} \operatorname{Atanh}\left(\theta_{2}-\theta_{3} n\right)\right]-\theta_{5}}{\exp \left[\theta_{1} \operatorname{Atanh}\left(\theta_{2}-\theta_{3} n\right)\right]+1}= \\
=-\theta_{4}\left(1+\theta_{5}\right) z^{*}+W \\
\theta_{1}=\frac{2(1-A)}{A \sqrt{4 A^{2}+1}}, \quad \theta_{2}=\frac{2 A-1}{\sqrt{4 A^{2}+1}}, \theta_{3}=\frac{4 A}{\sqrt{4 A^{2}+1}}
\end{gathered}
$$

$W, \theta_{4}$, and $\theta_{5}$ are integration constants. Eqn (7) is an interesting solution, but somewhat difficult to analyze for different values of the defined constants. Intending a more objective discussion, eqn (4) was integrated here furnishing a simpler form of the first order equation. The modified integration of the second order equation (4) leads to eqn (8). $K$ is an integration constant. $\theta_{l}$ are defined in eqn (7). Eqn (8) implies $\mathrm{d} n / \mathrm{d} z * \rightarrow 0$ for $n \rightarrow 0$, and admits exact solutions. In this study, exact solutions are shown for $\theta_{1} / 2=1,2$, and $1 / 2$, corresponding to $A=\sim 0.43099, \sim 0.30007$, and $\sim 0.56900$, respectively. Each $A$ leads to a particular explicit solution. But depending on $A$, the governing eqn (8) is only numerically integrable. Two additional numerical solutions, for $\theta_{1} / 2=3$ and $4(A \sim 0.23215$ and 0.18949 , respectively), are also shown.

$$
\frac{d n}{d z^{*}}=K\left(\frac{1+\theta_{2}}{1-\theta_{2}}\right)^{\theta_{1} / 2}\left[\left(\frac{1-\frac{\theta_{3} n}{1+\theta_{2} n}}{1+\frac{\theta_{3} n}{1+\theta_{2} n}}\right)^{\theta_{1} / 2}-1\right]
$$




\subsection{Solution of eqn (8) for $\theta_{1} / 2=1$, or $A \sim 0.43099$}

$\theta_{1} / 2=1$ is the simplest exponent in eqn (8). The solution is given by:

$$
z^{*}=\left\{\frac{n+\alpha_{2} \ln (n)+T_{2}}{\beta_{2}}\right\}, \text { with }\left\{\begin{array} { l } 
{ \theta _ { 1 } = 2 . 0 0 0 0 } \\
{ \theta _ { 2 } \sim - 0 . 1 0 4 5 4 , } \\
{ \theta _ { 3 } \sim 1 . 3 0 5 8 }
\end{array} \left\{\begin{array}{l}
\alpha_{2}=\frac{1-\theta_{2}}{\theta_{3}} \sim 0.84587 \\
\beta_{2}=\frac{2 K_{2}}{\theta_{2}-1} \sim-1.8107 K_{2}
\end{array}\right.\right.
$$

$T_{2}$ is an integration constant, and $\alpha_{2}$ and $\beta_{2}$ are auxiliary constants. The index 2 corresponds to $\theta_{1}=2$. For this value of $\theta_{1}, n$ may vary in the whole domain $0 \leq n \leq 1$. Using the boundary conditions: $n=0.01$ at $z^{*}=1$; and $n=1$ at $z^{*}=0$, it was obtained that $T_{2}=-1$ and $K_{2} \sim 2.6980$. Fig. 2(a) shows the graph of $n$. Fig. 2(b) shows that the modulus of the derivative $\mathrm{d} n / \mathrm{d} z^{*}$ always decreases with $z^{*}$.

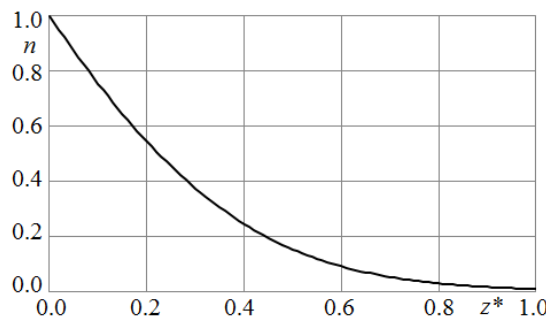

(a)

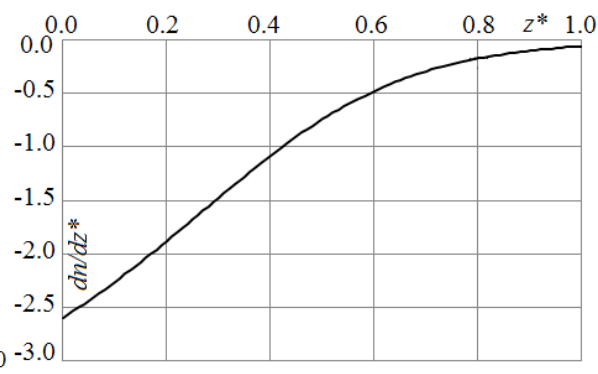

(b)

Figure 2: (a) Profile of $n$ (concentration) for $\theta_{1}=2$; (b) Profile of $\mathrm{d} n / \mathrm{d} z^{*}$.

\subsection{Solution of eqn (8) for $\theta_{1} / 2=2$, or $\mathrm{A} \sim 0.30007$}

The solution for $\theta_{1} / 2=2$ is given by eqn (10). Like in the former case, $T_{4}$ is an integration constant, and $\alpha_{4}, \beta_{4}, \gamma_{4}$ are auxiliary constants. Again, $n$ may vary in the whole domain $0 \leq n \leq 1$ for $\theta_{1}=4 . T_{4}$ and $K_{4}$ were obtained using $n=0.01$ at $z^{*}=1$; and $n=1$ at $z^{*}=0$, leading to $T_{4} \sim-3.3517$ and $K_{4} \sim 6.6779$. Figs. 3(a) and 3(b) show the profile of $n$ and evolution of $\mathrm{d} n / \mathrm{d} z^{*}$ with $z^{*}$, respectively.

$$
\begin{aligned}
& z^{*}=\left\{\frac{\frac{\alpha_{4}^{2}}{\beta_{4}} \ln (n)-\frac{\left(\alpha_{4}+\beta_{4}\right)^{2}}{\beta_{4}} \ln \left(\beta_{4}-n\right)-n-T_{4}}{-\gamma_{4}}\right\}, \text { with }\left\{\begin{array}{l}
\theta_{1}=4.0000 \\
\theta_{2} \sim-0.34285, \\
\theta_{3} \sim 1.0292
\end{array}\right. \\
& \alpha_{4}=\frac{1-\theta_{2}}{\theta_{3}} \sim 1.3048, \beta_{4}=\frac{1-\theta_{2}^{2}}{-\theta_{2} \theta_{3}} \sim 2.5009, \gamma_{4}=\frac{-4 K_{4} \theta_{2}}{\left(1-\theta_{2}\right)^{2}} \sim-0.76052 K_{4}
\end{aligned}
$$




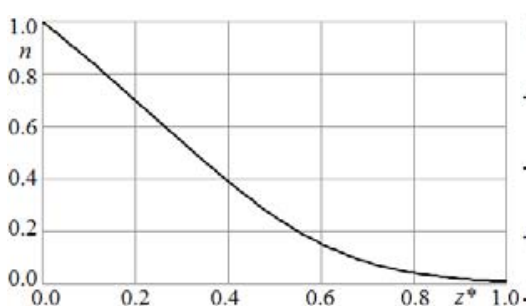

(a)

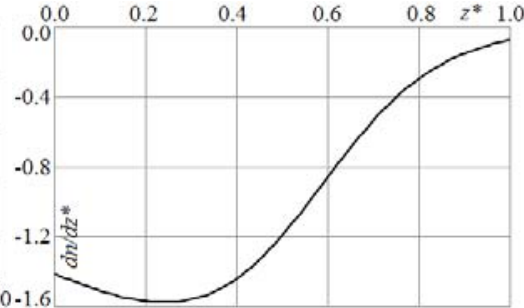

(b)

Figure 3: (a) Profile of $n$ (concentration) for $\theta_{1}=4$; (b) $\mathrm{d} n / \mathrm{d} z^{*}$ with a minimum.

Fig. 3(a) presents an inflexion, represented by the minimum of Fig. 3(b). That is, the largest concentration gradient (in modulus) does not occur at the interface. From eqn (8), following condition applies in the case of non-integer exponents $\theta_{1} / 2$ :

$$
n \leq \frac{1+\theta_{2}}{\theta_{3}}, \quad \text { or } \quad n \leq \frac{\sqrt{4 A^{2}+1}}{4 A}+\frac{2 A-1}{4 A}
$$

In the present case an integer exponent was used, but the minimum of Fig. 3(b) occurs at $n=\left(1+\theta_{2}\right) / \theta_{3} \sim 0,63852$, that is, at the equality point of eqn (11).

\subsection{Solution of eqn (8) for $\theta_{1} / 2=1 / 2$, or $A=\sim 0.56900$}

$\theta_{1} / 2=1 / 2$ was chosen to verify the behavior of solutions of non-integer exponents. Now $n$ may not be calculated for the whole domain $0 \leq n \leq 1$, because the limit of eqn (11) applies (to avoid roots of negative values in eqn (8)). The solution and $\theta_{l}$ for the present case are given by eqn (12). $T_{1}$ is an integration constant, and $\alpha_{1}, \beta_{1}$, $\gamma_{1}$ are auxiliary constants. In this case $n$ varies in the range $0 \leq n \leq\left[\sqrt{ }\left(4 A^{2}+1\right)+2 A\right.$ $1] / 4 A \sim 0.72625$ (see eqn 11). The values of $T_{1}, K_{1}$, and $Z_{L}$ were obtained using three boundary conditions: i) $n=0.01$ at $z^{*}=1.0$; ii) $n=\left[\sqrt{ }\left(4 A^{2}+1\right)+2 A-1\right] / 4 A$ at

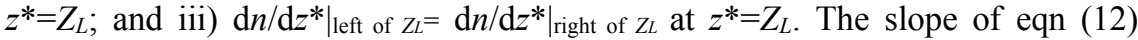
at $z^{*}=Z_{L}$ (right side) was equated to the slope of eqn (6) (left side of $z^{*}=Z_{L}$ ), because it is a solution of the original eqn (4). The obtained values are: $T_{1} \sim$ $0.74518 ; K_{1} \sim 3.1124$, and $Z_{L} \sim 0.080276$. In eqn (6) $\Omega_{2} \sim-3.4101$. Figs. 4 (a) and 4(b) show the obtained results for $n$ and $\mathrm{d} n / \mathrm{d} z^{*}$, respectively.

$$
\begin{aligned}
& z^{*}=\frac{\left\{\begin{array}{l}
\sqrt{1+\left(\beta_{1}-\alpha_{1}\right) n-\alpha_{1} \beta_{1} n^{2}}-\frac{\beta_{1}-\alpha_{1}}{2 \sqrt{\alpha_{1} \beta_{1}}} \operatorname{Asin}\left(\frac{\beta_{1}-\alpha_{1}-2 \alpha_{1} \beta_{1} n}{\alpha_{1}+\beta_{1}}\right)- \\
-\ln \left[2 \sqrt{1+\left(\beta_{1}-\alpha_{1}\right) n-\alpha_{1} \beta_{1} n^{2}}+\left(\beta_{1}-\alpha_{1}\right) n+2\right]+2 \ln (n)+\beta_{1} n-T_{1}
\end{array}\right\}}{-\gamma_{1}\left(\alpha_{1}+\beta_{1}\right)} \\
& \text { with }\left\{\begin{array} { l } 
{ \theta _ { 1 } = 1 . 0 0 0 0 } \\
{ \theta _ { 2 } \sim 0 . 0 9 1 0 9 3 , } \\
{ \theta _ { 3 } \sim 1 . 5 0 2 4 }
\end{array} \quad \left\{\begin{array}{l}
\alpha_{1}=\theta_{3} /\left(1+\theta_{2}\right) \sim 1.3769 \\
\beta_{1}=\theta_{3} /\left(1-\theta_{2}\right) \sim 1.6529 \\
\gamma_{1}=K\left[\left(1+\theta_{2}\right) /\left(1-\theta_{2}\right)\right]^{1 / 2} \sim 1.0957 K
\end{array}\right.\right.
\end{aligned}
$$




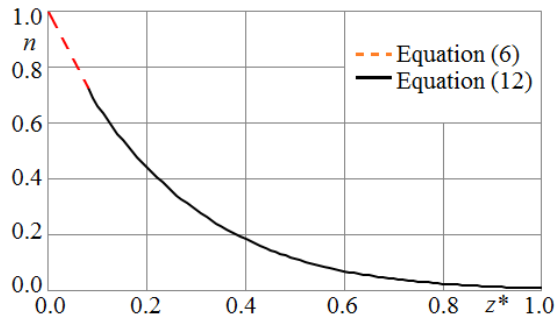

(a)

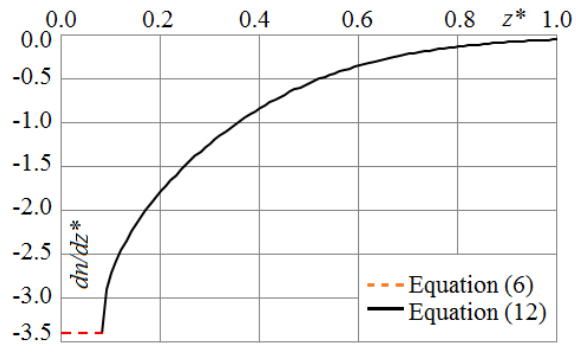

(b)

Figure 4: (a) Composed profiles of $n$ (scalar concentration in the flow) for $\theta_{1}=1$; (b) $\mathrm{d} n / \mathrm{d} z^{*}$ showing the slopes of eqns (6) and (12).

Table 1 reproduces the equations that compose the solution of this case, and their interval of application for $n$. Both equations satisfy the governing eqn (4).

Table 1: Equations of $n$ for $\theta_{1} / 2=1 / 2$ and $A \sim 0.56900$.

\begin{tabular}{|c|c|}
\hline Interval of $n$ & Equation \\
\hline $\begin{array}{c}\frac{\sqrt{4 A^{2}+1}}{4 A}+\frac{2 A-1}{4 A} \\
\leq n \leq 1\end{array}$ & $n=-\Omega_{2} z^{*}+1$ \\
\hline $0 \leq n \leq$ & $z^{*}=$ \\
$\frac{\sqrt{4 A^{2}+1}}{4 A}+\frac{2 A-1}{4 A}$ & $\left\{\begin{array}{l}\sqrt{1+\left(\beta_{1}-\alpha_{1}\right) n-\alpha_{1} \beta_{1} n^{2}}-\frac{\beta_{1}-\alpha_{1}}{2 \sqrt{\alpha_{1} \beta_{1}}} \operatorname{Asin}\left(\frac{\beta_{1}-\alpha_{1}-2 \alpha_{1} \beta_{1} n}{\alpha_{1}+\beta_{1}}\right)- \\
-\ln \left[2 \sqrt{1+\left(\beta_{1}-\alpha_{1}\right) n-\alpha_{1} \beta_{1} n^{2}}+\left(\beta_{1}-\alpha_{1}\right) n+2\right]+2 \ln (n)+\beta_{1} n-T_{1}\end{array}\right\}$ \\
$-\gamma_{1}\left(\alpha_{1}+\beta_{1}\right)$ \\
\hline
\end{tabular}

\subsection{Joint analysis}

Figs. 5(a) and 5(b) show all profiles of $n$ and $\mathrm{d} n / \mathrm{d} z^{*}$ plotted together, also with numerical solutions for $\theta_{1} / 2=3$ and 4 , or $A \sim 0.23215$ and 0.18949 , respectively.

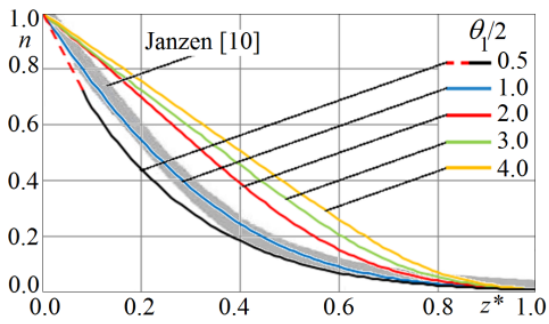

(a)

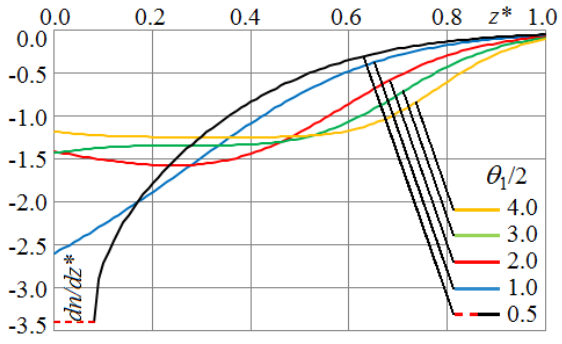

(b)

Figure 5: (a) Profiles of $n$ (scalar concentration in the flow). The gray cloud represents experimental data of Janzen [10]; (b) Profiles of $\mathrm{d} n / \mathrm{d} z^{*}$. 
Fig. 5 shows that higher $\theta_{1} / 2$ impose "low varying" $\mathrm{d} n / \mathrm{d} z *$ close to the surface (curves for $\theta_{1} / 2=2,3$, and 4). High $\theta_{1} / 2$ (or low $A$ ) reflect high diffusion effects, according to Schulz et al. [26]. For non-integer $\theta_{1} / 2$ (exemplified with $\theta_{1} / 2=1 / 2$ ), $\mathrm{d} n / \mathrm{d} z^{*}$ at the surface is taken as the slope of the linear solution of the governing eqn (4). Both behaviors agree with the practical procedure of adopting a linear profile of the mass concentration close to the surface, but also solutions with varying $\mathrm{d} n / \mathrm{d} z^{*}$ were obtained, as shown by the profile for $\theta_{1} / 2=1$. All solutions were obtained for constant values of the reduction function $(\alpha=1-A)$. The existence of inflection points may be a consequence of using the constant $\alpha$ values. Eqn (8) leads to solutions compatible with observations. The data of Janzen [10] superpose better with the curve for $\theta_{1} / 2=1$.

\section{Conclusions}

Theoretical and numerical profiles for scalar variables in interfacial turbulent gas-liquid transfer were presented. It was found that the slope of the profiles close to interfaces may decrease continuously (in modulus) or maintain an approximate constant value, depending on the value of the reduction function $\alpha$ of the RSW method (replaced by $A$ or the equivalent $\theta_{1} / 2$ in this study), related to the level of agitation of the liquid. Integer and non-integer values of $\theta_{1} / 2$ may be used. Single solutions and composition of different solutions of the governing equation were used to cover the whole boundary layer domain, depending on $\theta_{1} / 2$. The solutions furnished behaviors compatible with measured profiles found in the literature.

\section{Appendix A}

RSW eqns (A1), (A2), and (A3), reproduced from eqns (48a, b) and (49) of Schulz et al. [26], allow obtaining eqn (2) of the text.

$$
\begin{gathered}
1-n=\frac{1}{\kappa} \frac{d^{2} n}{d z^{*}}-\frac{d\left(I J^{*}\right)}{d z^{*}} \\
-n(1-n) A^{2}+I J^{*} \frac{d n}{d z^{*}}+\frac{A}{2} \frac{d\left[I J^{*}(1-2 n)\right]}{d z^{*}}=\frac{-2}{\kappa} n(1-n) A^{2} \frac{d^{2} n}{d z^{* 2}} \\
I J^{*}=n(1-n) A \sqrt{w^{2}} /\left[Y E \sqrt{n(1-n)+\beta(1-\beta) /(2 \beta-1)^{2}}\right]
\end{gathered}
$$

The integration of eqn (A1) produces eqn (A4). Combining it algebraically with eqns (A1) and (A2) leads to eqn (A5), which is eqn (2) of the text.

$$
I J^{*}=\frac{1}{\kappa} \frac{d n}{d z^{*}}-z^{*}+\int n d z^{*}+C
$$




$$
\begin{aligned}
& -A(1-n)\left[A n+\frac{1+2 n}{2}\right]+\left[\frac{1}{\kappa} \frac{d n}{d z^{*}}-z^{*}+\int n d z *+C\right](1-A) \frac{d n}{d z^{*}}= \\
& =\frac{-A}{2 \kappa}[4 A n(1-n)+1-2 n] \frac{d^{2} n}{d z^{2}}
\end{aligned}
$$

$C$ is an integration constant. $I J^{*}$ is related to ${ }_{\mathrm{w}^{2}}$, the rms velocity. $Y$ is the scalar transfer coefficient. $\beta$ quantifies the superposition of scalar and velocity records.

\section{Appendix B}

Eqns (A1) and (A2) easily transform to (B1) and (B2) for stationary turbulence (see Gonçalves [30] and Schulz et al. [26]), used to obtain eqn (3) of the text.

$$
\begin{gathered}
0=\frac{1}{\kappa} \frac{d^{2} n}{d z^{2}}-\frac{d\left(I J^{*}\right)}{d z^{*}} \\
I J * \frac{d n}{d z^{*}}+\frac{A}{2} \frac{d\left[I J^{*}(1-2 n)\right]}{d z^{*}}=\frac{-2}{\kappa} n(1-n) A^{2} \frac{d^{2} n}{d z^{* 2}}
\end{gathered}
$$

Integrating eqn (B1) produces eqn (B3). Combining it with eqns (B1) and (B2) leads to eqn (B4), which is eqn (3). $C_{G}$ is an integration constant and $\Omega_{2}=\kappa C_{G}$.

$$
\begin{gathered}
I J^{*}=\left(d n / d z^{*}\right) / \kappa+C_{G} \\
(1-A)\left[\frac{d n}{d z^{*}}+\Omega_{2}\right] \frac{d n}{d z^{*}}=\frac{-A}{2}[4 A n(1-n)+1-2 n] \frac{d^{2} n}{d z^{* 2}}
\end{gathered}
$$

\section{References}

[1] Asher, W.E., Liang, H., Zappa, C.J., Loewen, M.R., Mukto, M.A., Lichtendorf, T.M. \& Jessup, A.T., Statistics of surface divergence and their relation to air-water gas transfer velocity, Journal of Geophysical Research: Oceans, 17(C5), pp. 1-15, 2012.

[2] Demars, B.O.L. \& Manson, J.R., Temperature dependence of stream aeration coefficients and the effect of water turbulence: a critical review, Water Research, 47(1), pp. 1-15, 2013.

[3] Nguyen, M.T. \& Tan, S.K., Effect of waves on reaeration, AIChE Journal, 59(12), pp. 4839-4845, 2013.

[4] Krah, N., Visualization of air and water-sided concentration profiles in laboratory gas exchange experiments, PhD Thesis, U. of Heidelberg, 2014.

[5] Gualtieri, C. \& Doria, P., Gas transfer at unsheared free-surfaces (Chapter 6). Fluid mechanics of environmental interfaces, eds. C. Gualtieri, \& D.T. Mihailovic, CRC-Taylor Francis-Balkema, pp. 145-179, 2012.

[6] Nagaosa, R. \& Handler, R.A., Characteristic time scales for predicting the scalar flux at a free surface in turbulent open-channel flows, AIChE Journal, 58(12), pp. 3867-3877, 2012. 
[7] Janzen, J.G., Schulz, H.E. \& Jirka, G., Turbulent gas flux measurements near the air-water interface in an oscillating-grid tank, Gas Transfer at Water Surfaces, eds. S. Komori, W. McGillis \& R. Kurose, Kyoto U. Press, Kyoto, pp. 65-77, 2011.

[8] Schulz, H.E. \& Janzen, J.G., Concentration fields near air-water interfaces during interfacial mass-transport: oxygen transport and random square wave analysis, Brazilian J. of Chemical Engineering, 26(3), pp. 527-536, 2009.

[9] Gonzales, B.C.C., Lamon, A.W., Janzen, J.G., Campos, J.R. \& Schulz, H.E., Microscopic sensors for oxygen measurements at air-water interfaces and sediment biofilms, Gas Transfer at Water Surfaces, eds. S. Komori, W. McGillis \& R. Kurose, Kyoto U. Press, Kyoto, pp. 507-515, 2011.

[10] Janzen, G.J., Gas transfer near the air-water interface in oscillating-grid tanks and properties of isotropic turbulent flows - text in Portuguese, $\mathrm{PhD}$ thesis, U. of São Paulo, Brazil, 2006.

[11] Wolff, L.M., Liu, Z.C. \& Hanratty, T.J., A fluorescence technique to measure concentration gradients near an interface, Air-Water Mass Transfer, eds. S.C. Wilhelms \& J.S. Gulliver, ASCE, New York, pp. 210 218, 1991.

[12] Duke, S.R. \& Hanratty, T.J., Measurements of the concentration field resulting from oxygen absorption at a wavy air-water interface, Air-Water Gas Transfer, eds: B. Jähne \& E.C. Monahan, pp. 627-635, 1995.

[13] Münsterer, T., Mayer, H.J. \& Jähne, B., Dual-tracer measurements of concentration profiles in the aqueous mass boundary layer, Air-Water Gas Transfer, eds. B. Jähne \& E.C. Monahan, pp. 637-648, 1995.

[14] Woodrow, P.T. \& Duke S.R., LIF measurements of oxygen concentration gradients along flat and wavy air-water interfaces, Gas Transfer at Water Surfaces, ed. M.A. Donelan, W.M. Drennan, E.S. Saltzman \& R. Wanninkhof, Geophysical Monograph Series, 127, pp. 83-88, 2002.

[15] Falkenroth, A., Visualisation of oxygen concentration profiles in the aqueous boundary layer, $\mathrm{PhD}$ thesis, Ruperto-Carola U. of Heidelberg, 2006.

[16] Calmet, I. \& Magnaudet, J., High-Schmidt number mass transfer through turbulent gas-liquid interfaces, International Journal of Heat and Fluid Flow, 19(5), pp. 522-532, 1998.

[17] Hasegawa, Y. \& Kasagi, N., The effect of Schmidt number on air-water interface mass transfer, Proc. 4th Int. Conference on Multiphase Flow, New Orleans, pp. 1-11, 2001.

[18] Hasegawa, Y. \& Kasagi, N., Hybrid DNS/LES of high Schmidt number mass transfer across turbulent air-water interface. International J. of Heat and Mass Transfer, 52 (3/4), pp. 1012-1022, 2009.

[19] Calmet, I. \& Magnaudet, J., Statistical structure of high-Reynolds-number turbulence close to the free surface of an open-channel flow, J. of Fluid Mechanics, 474, pp. 355-378, 2003.

[20] Magnaudet, J. \& Calmet, I., Turbulent mass transfer through a flat shearfree surface, J. of Fluid Mechanics, 553, pp. 155-185, 2006. 
[21] Ishihara, T., Hunt, J.C.R. \& Kaneda, Y., Conditional analysis near strong shear layers in DNS of isotropic turbulence at high Reynolds number, $13^{\text {th }}$ European Turbulence Conference, J. of Physics Conference Series, 318, paper 042004, 2011.

[22] Hinze, J.O., Turbulence, McGraw-Hill Book Company, USA, 1959.

[23] Brodkey, R.S., The phenomena of fluid motions, Addison-Wesley Publishing Company, Massachusetts, 1967.

[24] Monin, A.S. \& Yaglom, A.M., Statistical Fluid Mechanics: Mechanics of Turbulence, Vols. 1 and 2, the MIT Press, 1981.

[25] Poper, S.B., Turbulent Flows, Cambridge U. Press, 2000.

[26] Schulz, H.E., Lopes Jr., G.B., Simões, A.L.A. \& Lobosco, R.J., Onedimensional turbulent transfer using random square waves - scalar/velocity and velocity/velocity interactions (Chapter 1). Hydrodynamics: Advanced Topics, eds. H.E. Schulz, A.L.A. Simões \& R.J. Lobosco, InTech, Rijeka, pp. 3-34, 2011.

[27] Schulz, H.E., Simões, A.L.A. \& Janzen, J.G., Statistical approximations in gas-liquid mass transfer, Gas Transfer at Water Surfaces, eds S. Komori, W. McGillis \& R. Kurose, Kyoto U. Press, Kyoto, pp. 208-221, 2011.

[28] Lopes Jr., G.B. \& Schulz, H.E., Analyses of boundary conditions for onedimensional mass transfer in turbulent flow - text in Portuguese, $24^{\text {th }}$ National Congress of Applied and Computational Mathematics, Brazil, pp. 391-397, 2012.

[29] Gonçalves, B.B. \& Schulz, H.E., One-dimensional turbulent mass transfer at air-water interfaces: details of discontinuities of derivatives using the RSW method, Computational Methods in Multiphase Flow VII, eds. C.A. Brebbia, \& P. Vorobieff, WIT Press, pp. 365-376, 2013.

[30] Gonçalves, B.B., Detailing the one-dimensional solution for the RSW's method considering a constant reduction function applied to the turbulent interfacial scalar transport - text in Portuguese. MSc. Dissertation, U. of São Paulo, Brazil, 2014.

[31] Souza, M.V., Kasnodzei, P.E.A. \& Schulz, H.E., Theoretical/numerical study of RSW as a statistical tool for transport in turbulent flows: $2^{\text {nd }}$ order governing equation - text in Portuguese), $21^{\text {st }}$ USP International Symposium on Scientific Initiation, Paper 1701, São Carlos, 2013.

[32] Lewis, W.K \& Withman, W.G., Principles of gas absorption, Ind. Engg. Chem., 16, pp. 1215-1220, 1924. 